\title{
Avaliação do tratamento de fissura anal crônica com isossorbida tópica a $1 \%$
}

\author{
Evaluation of the treatment of chronic anal fissure with topical isosorbide $1 \%$
}

\author{
ANA CAROLINA LISBOA PRUDENTE ${ }^{1}$, VALDINALDO ARAGÃO DE MELO ${ }^{2}$, JUVENAL DA ROCHA TORRES \\ $\mathrm{NETO}^{3}$, RODRIGO ROCHA SANTIAGO ${ }^{4}$, MÁRIO AUGUSTO DO NASCIMENTO VIDAL ${ }^{5}$
}

\begin{abstract}
${ }^{1}$ Mestranda em Ciências da Saúde pela Universidade Federal de Sergipe (UFS) - Aracaju (SE), Brasil.
${ }^{2}$ Professor Doutor em Cirurgia do Aparelho Digestivo da UFS - Aracaju (SE), Brasil. ${ }^{3}$ Professor Doutor em Cirurgia

do Aparelho Digestivo da UFS - Aracaju (SE), Brasil. ${ }^{4}$ Médico especialista em Coloproctologia pela Associação

Médica Brasileira; Preceptor em Residência Médica de Coloproctologia da UFS - Aracaju (SE), Brasil.

${ }^{5}$ Médico Coloproctologista - Aracaju (SE), Brasil.
\end{abstract}

PRUDENTE ACL, MELO VA, TORRES NETO JR, SANTIAGO RR, VIDAL MAN. Avaliação do tratamento de fissura anal crônica com isossorbida tópica a 1\%. Rev bras Coloproct, 2011;30(4): 409-413.

RESUMO: Introdução: Fissuras anais crônicas são úlceras benignas, dolorosas, profundas. Ocorrem devido a trauma das fezes, hipertonia esfincteriana e pobre vascularização. Cirurgia é mais efetiva, porém com efeitos adversos (incontinência anal). Terapia conservadora consegue decréscimo transitório da pressão de repouso, cicatrizando muitas lesões, sem dano muscular. Métodos: Objetivando avaliar tratamento de fissuras crônicas com isossorbida (ISO) a 1\% tópica, foi realizado um ensaio clínico, duplo-cego em pacientes do Serviço de Coloproctologia da Universidade Federal de Sergipe (UFS) - Aracaju, Sergipe, durante um ano. Foram estudados 24 pacientes: 14 no Grupo 1 - creme com ISO, e 10 no Grupo 2 - placebo. Avaliaram-se comportamento da pressão de repouso, melhora da dor e grau de cicatrização das feridas com e sem ISO. Resultados: Resultados mostraram que a fissura acometeu mais mulheres, a constipação foi observada em 58,3\%. Quanto à dor, obteve-se menor intensidade no Grupo 2, mas sem significância. A cicatrização ao fim de 60 dias foi igual nos dois grupos (50\%). Quanto às médias de pressão de repouso com 30 e 60 dias, houve queda no padrão em ambos os grupos, porém sem significância. Observou-se que pacientes curados foram os de maior redução de pressão de repouso. Conclusão: Concluiu-se que a ISO não modificou o padrão de resposta manométrica; todavia, houve melhora clínica importante nos dois grupos, cuja taxa de cicatrização foi equivalente.

Descritores: Fissura anal. Isossorbida.

\section{INTRODUÇÃO}

As fissuras anais são patologias anorretais bastante frequentes na população. São úlceras benignas, na maioria das vezes traumáticas pela constipação do paciente, porém podem causar grandes transtornos na atividade diária de seu portador. Seu tratamento é cirúrgico e envolve a secção da musculatura esfincteriana interna do ânus. Terapias conservadoras com medicações tópicas são utilizadas com o objetivo de relaxar essa musculatura anal de forma temporária, possibili- tando a cicatrização da fissura. Este estudo foi direcionado no sentido de escolher a isossorbida (ISO) como agente químico.

\section{OBJETIVO}

Avaliar a influência da terapia com ISO 1\% na variação de pressão de repouso do canal anal, o seu efeito na melhora clínica de portadores de fissura anal, e verificar a relação da variação da PREP com a taxa de cicatrização da lesão. 


\section{MATERIAIS E MÉTODOS}

Ensaio clínico duplo-cego realizado no Hospital Universitário da Universidade Federal de Sergipe (UFS), no período de abril de 2008 a março de 2009 , com pacientes portadores de fissura anal crônica. A pesquisa foi aceita pelo Comitê de Ética em Pesquisa. Totalizou 24 pacientes que foram divididos em dois grupos: Grupo 1 - uso de creme com agente ativo (ISO 1\%) - 14 pacientes; Grupo 2 - uso de creme sem agente ativo -10 pacientes. Critérios de exclusão: úlceras atípicas e agudas, doenças cirúrgicas concomitantes, cirurgias prévias. Randomização: cremes codificados pela farmácia de manipulação sem o conhecimento da equipe. Entrega aleatória após a primeira manometria. Preenchimento do protocolo e orientação dos pacientes quanto ao uso de cremes ( 3 vezes/dia) e à dieta/higiene. O seguimento ambulatorial dos pacientes foram 8,15 e 30 dias no primeiro mês, 45 e 60 dias, no segundo. A manometria foi repetida com 30 e 60 dias do



口Feminino

口Masculino

Figura 1 - Distribuição dos pacientes quanto ao gênero

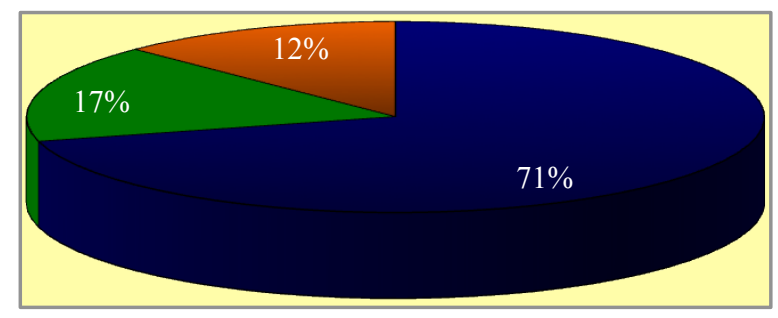

- Posterior

口Anterior/posterior

$\square$ Anterior

Figura 2 - Localização das fissuras uso do creme. Parâmetros avaliados: caracterização da amostra, apresentação clínica inicial, localização das fissuras anais, intensidade da dor e grau de cicatrização das lesões com uso dos cremes em 30 e 60 dias, perfil da pressão de repouso antes e após a instituição da terapia. Análise estatística iniciou-se com organização dos dados em planilhas do Microsoft Excel e foi finalizada com análise dos dados em SPSS 15.

\section{RESULTADOS}

O estudo contemplou um total de 24 pacientes, sendo $14(58,3 \%)$ pertencentes ao Grupo 1, que utilizou a ISO, e 10 (41,7\%), ao Grupo 2, que usou o placebo.

A idade variou de 21 a 69 anos, com média de 34,8 anos $( \pm 10,4$ anos $)$.

Quanto ao gênero, houve um predomínio de $83 \%$ de mulheres acometidas por fissura anal crônica $(\mathrm{p}=0,002)$ (Figura 1).

Quanto à apresentação clínica inicial, a dor foi sintoma mais prevalente em ambos os grupos, atingindo $100 \%$ dos casos no Grupo 1 e 95,8\%, no Grupo 2. O sangramento retal esteve presente em $71,5 \%$ dos casos no Grupo 1, enquanto a sensação de tumoração foi mais comum nos pacientes do Grupo 2 (80\%). Avaliando o ritmo intestinal, a constipação foi avaliada em $58,7 \%$ dos casos (Tabela 1).

Considerando a localização das fissuras, constatou-se sua predominância na comissura posterior: $70 \%$ dos casos (Figura 2). As fissuras anteriores acometeram $20,8 \%$ dos indivíduos, e desses, $71,4 \%$ eram mulheres.

No tocante à dor durante a evacuação, após 60 dias de tratamento o padrão de intensidade no Grupo 2 foi menor (40\%), variando a nota de 1 a 2, considerada como dor leve e ocasional. Já no Grupo 1, 50\% dos pacientes referiram dor leve e $14 \%$ dor moderada, atribuindo notas 6 e 7 na escala visual. Não há diferença estatística entre os grupos $(p=0,49)$.

Tabela 1 - Apresentação clínica inicial

\begin{tabular}{lccc}
\hline & $\begin{array}{c}\text { Grupo 1 } \\
\mathbf{n}=\mathbf{1 4}(\mathbf{\%})\end{array}$ & $\begin{array}{c}\text { Grupo 2 } \\
\mathbf{n = 1 0}(\mathbf{\%})\end{array}$ & Total \\
\hline Dor & $14(100)$ & $9(90)$ & $23(95,8)$ \\
Hematoquesia & $10(71,1)$ & $6(60)$ & $16(66,6)$ \\
Tumor & $8(57,1)$ & $8(80)$ & $16(66,6)$ \\
Constipação & $9(64,3)$ & $5(50)$ & $14(58,3)$ \\
\hline
\end{tabular}


Estratificando-se a taxa de cicatrização por grupos de cremes, obteve-se que ao final de 60 dias, metade $(50 \%)$ dos pacientes de cada grupo havia cicatrizado suas lesões (Tabela 2).

Quanto ao perfil das médias das pressões de repouso no intervalo de 30 e 60 dias, constatou-se ao fim do estudo, uma redução pressórica em ambos os grupos, porém sem significância estatística (Tabela 3).

Fazendo-se a relação entre o perfil pressórico e a ocorrência da cicatrização da fissura, observou-se que a média da pressão de repouso aos 30 e 60 dias foi menor nos pacientes em que houve cicatrização, independentemente do uso ou não da ISO $(p=0,24)$ (Figuras 3 e 4 ).

\section{DISCUSSÃO}

No estudo em questão, a idade da amostra variou de 21 a 69 anos, com média de 34,8 anos, semelhante ao estudo de Pinho et al. ${ }^{1}$ que envolveu 256 pacientes, com média de idade de 32 anos. Percebe-se na literatura que as fissuras anais acometem mais as mulheres, como foi visto por Lysy et al. ${ }^{2}(57,4 \%)$. Pinho et al. ${ }^{1}$ obtiveram um índice mais elevado com $68 \%$ dos casos. Na pesquisa atual, houve uma supremacia ainda maior do gênero feminino $(83 \%)$. Esses números se devem à maior procura de mulheres ao consultório médico, assim como é sabido da maior prevalência de doenças anorretais nesse gênero.

A dor anal evacuatória é o principal sintoma na doença fissurária ${ }^{3}$ seguida pelo sangramento retal. A dor esteve presente em $95,8 \%$ dos casos, enquanto a hematoquesia, em $66,6 \%$ deles. Pascual et al. observaram a dor anal em $82 \%$ dos seus pacientes e retorragia em $40 \%{ }^{4}$. Quanto à presença de plicomas anais, encontrou-se a taxa de $66,6 \%$ de casos, bem superior à casuística de Pascual et al. ${ }^{1}$, de $22 \%$. Isso pode ser explicado porque esta pesquisa contemplou apenas casos de fissura crônica.

A constipação é um fator importante na gênese da fissura anal ${ }^{5}$. Em muitos estudos, a constipação foi apontada com frequência, no entanto, essa ligação não é tão estreita. Pascual et al. ${ }^{4}$ observaram $28 \%$ de constipados em sua amostra. No presente estudo, 58,3\% dos pacientes eram constipados.

A localização posterior também é um ponto interessante. A amostra revelou que $70 \%$ das lesões en-
Tabela 2 - Taxa de cicatrização por grupos

\begin{tabular}{llc}
\hline & $\begin{array}{c}\text { Cicatrização } \\
\mathbf{3 0} \text { dias }\end{array}$ & $\begin{array}{c}\text { Cicatrização } \\
\mathbf{6 0} \text { dias }\end{array}$ \\
\hline Grupo 1 & $2(14,3 \%)$ & $7(50 \%)$ \\
Grupo 2 & $1(10 \%)$ & $5(50 \%)$ \\
\hline
\end{tabular}

Tabela 3 - Médias de pressão de repouso em três momentos

\begin{tabular}{lcc}
\hline & Grupo 1 & Grupo 2 \\
\hline PREP inicial & $112,36 \mathrm{mmHg}$ & $105,9 \mathrm{mmHg}$ \\
(normal - 50 a 80 mmhg) & $(90-155 \mathrm{mmHg})$ & $(64-144 \mathrm{mmHg})$ \\
PREP 30 dias & $114,21 \mathrm{mmHg}$ & $99,2 \mathrm{mmHg}$ \\
& $(67-192 \mathrm{mmHg})$ & $(64-142 \mathrm{mmHg})$ \\
PREP 60 dias & $109,57 \mathrm{mmHg}$ & $98,4 \mathrm{mmHg}$ \\
& $(65-155 \mathrm{mmHg})$ & $(66-147 \mathrm{mmHg})$ \\
\hline
\end{tabular}

PREP: pressão de repouso.

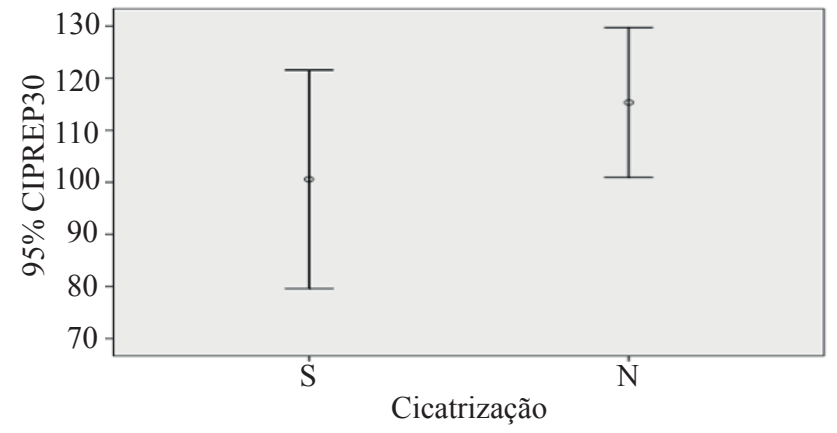

Figura 3 - Média das pressões de repouso - 30 dias para os grupos com e sem cicatrização

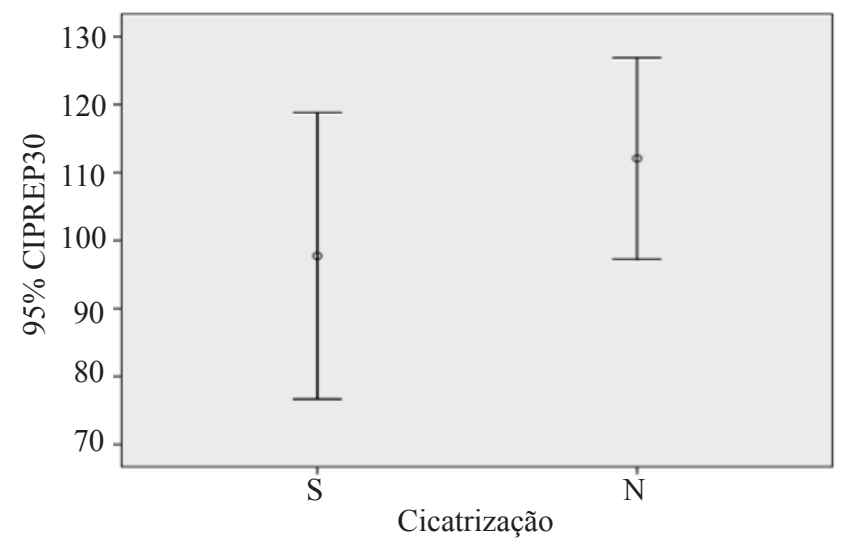

Figura 4 - Média das pressões de repouso - 60 dias para os grupos com e sem cicatrização 
contravam-se posteriormente e $17 \%$, anteriormente. Nas mulheres, a fissura anterior esteve presente em $20,8 \%$ dos casos. Pascual et al. ${ }^{4}$ encontraram $65 \%$ das feridas na região posterior e $24 \%$ na anterior. Lysy et al. ${ }^{2}$ confirmaram esses números com casuística maior: $68,4 \%$ posterior, $21,3 \%$ anterior, $7,7 \%$ mais de uma lesão. Esses autores viram 14\% de fissuras anteriores em mulheres. Explica-se esse fato por detalhes anatômicos, em que a configuração elíptica da musculatura do canal anal tornava mais frágil e suscetível as zonas anterior e posterior, por ser menor a quantidade de fibras de suporte ${ }^{4}$. A anatomia esfincteriana foi bem estudada pela ultrassonografia endoanal tridimensional ${ }^{6}$. Conheceu-se um espaço anterior no canal anal superior que não possui musculatura. Além disso, posteriormente, a musculatura é mais delgada, sendo, portanto, zonas de fragilidade ao trauma.

A melhora clínica, verificada pelo alívio da dor evacuatória, foi importante em ambos os grupos da atual pesquisa, com 40 a $50 \%$ dos casos considerando-a como dor leve e ocasional. Mesquita et al. ${ }^{7}$ demonstraram $84,6 \%$ de melhora clínica com terapia conservadora.

A taxa de cicatrização foi de $50 \%$ para os 2 grupos ao fim de 60 dias. Esse resultado condiz com a literatura. A cicatrização da ferida foi inferior aos trabalhos publicados como o de Mesquita et al. ${ }^{7}$, que apresentaram $77 \%$ de cura.

A manometria anorretal é utilizada para avaliar funcionalidade do esfíncter. No estudo de fissura anal, pressão de repouso é a medida mais pertinente por caracterizar o estado de tonicidade do esfíncter anal interno. Jones et al. ${ }^{8}$ concluíram que o número de pacientes com fissura anal crônica sem elevação do tônus esfincteriano é maior do que prevê o exame físico. A redução da pressão de repouso é esperada ao se optar pela terapia conservadora. Na pesquisa em questão, observou-se uma diminuição discreta nos níveis pressóricos, porém sem significância estatística. Mesquita et al. ${ }^{7}$ não perceberam significativa redução pressórica. Já Parellada ${ }^{9}$ conseguiu $89 \%$ de cura após 10 semanas com redução de $30 \%$ da pressão de repouso. Os números variam muito em cada trabalho publicado; no entanto, o mais essencial é que há pacientes que se curam com agentes tópicos e protegem sua musculatura esfincteriana.

Esta pesquisa comparou ainda pacientes com e sem cicatrização e o comportamento pressórico em cada um deles. Como condizente com a literatura, as lesões que persistiam foram aquelas em que o tônus esfincteriano se mantinha alto, tanto em 30 quanto em 60 dias.

\section{CONCLUSÃO}

Houve melhora clínica importante nos grupos com e sem ISO, especialmente no tocante à dor evacuatória.

A utilização da ISO 1\% não interferiu na cicatrização de fissuras anais. A taxa de cicatrização das feridas foi igual em ambos os grupos.

A utilização da ISO 1\% não modificou, significantemente, o padrão de resposta manométrica no tocante à pressão de repouso dos indivíduos com fissura anal crônica.

O decréscimo da pressão de repouso ocorreu nos dois grupos, sem diferença estatística; no entanto, foi mais acentuado no grupo dos pacientes em que as fissuras anais se cicatrizaram.

\footnotetext{
ABSTRACT: Introduction: Chronic anal fissures are deep, benign and painful ulcers. The involved factors are local trauma, sphincter hypertonia and poor blood flow. Surgery is gold standard treatment, but it causes fecal incontinence. Medical non-surgical therapy gets transitory resting pressure reduction and cure of lesions, without muscular damage. Methods: In order to evaluate the treatment of chronic anal fissures using topical isossorbide (ISO) $1 \%$, a randomizated and double-blind study twas carried out in Coloproctology Section of Universidade Federal de Sergipe (UFS), Sergipe, Brazil, during one year. Twenty-four patients were evaluated: 14 in Group 1 - ISO cream, and 10 in Group 2 - placebo. Resting pressure profile, improvement of painful symptoms and degree of scaring of the fissure were evaluated. Results: Ulcer was more prevalent in women, constipation was present in $58.3 \%$. The evacuatory pain was less common in Group 2, but without statistical significance. After 60 days, the healing was equal in the both groups (50\%). There was a small reduction of resting pressure means at the end of 30 and 60 days, without statistical significance. Healing patients presented more resting pressure reducing. Conclusion: ISO cream did not influence the manometric response standard; otherwise it offered clinical improvement in both groups, whose scarring index was similar.
}

Keywords: Fissure in ano. Isosorbide. 


\section{REFERÊNCIAS}

1. Pinho MSL; Ferreira LC; Vasconcelos ECG; Souza Filho NA; Reis MC. Análise da prevalência por sexo e idade nas doenças anorretais frequentes. Rev Bras Coloproct. 2002;3:158-63.

2. Lysy J, Israeli E, Levy S, Rozentzweig G, Strauss-Liviatan N, Goldin E. Long-term results of "chemical sphincterotomy" for chronic anal fissure: a prospective study. Dis Colon Rectum. 2006;49(6):858-64.

3. Corman M, Allison AJ, Kulhne JP. Manual de cirurgia colorretal. Rio de Janeiro: Revinter; 2006. p. 145-58.

4. Pascual M, Courtier R, Gil MJ et al. Estudio ecográfico y manométrico del esfíncter anal interno em indivíduos com fisura anal crônica. Cir Esp. 2005;77(1):27-30.

5. Cruz GMG. Coloproctologia: propedêutica nosológica. Rio de Janeiro: Revinter; 2000. v. II, p. 1157-66.

6. Regadas FSP, Regadas SMM. Distúrbios funcionais do assoalho pélvico. Rio de Janeiro: Revinter; 2007. p. 12-7.
7. Mesquita RM, Ramos JR, Gama JAC. Tratamento da fissura anal com dinitrato de isossorbida tópico. Experiência inicial. Rev Bras Coloproct. 1997;17(2):93-5.

8. Jones OM, Ramalingam T, Lindsey I, Cunningham C, George BD, Mortensen NJ. Digital rectal examination of sphincter pressures in chronic anal fissure is unreliable. Dis Colon Rectu; 2005;48:349-52.

9. Parellada C. Randomized, prospective trial comparing $0.2 \%$ isosorbide dinitrate ointment with sphincterotomy in treatment of chronic anal fissure: a two-year follow-up. Dis Colon Rectum. 2004;47(4):437-43.

Endereço para correspondência:

Ana Carolina Lisboa

Avenida Francisco Porto, 239, apto. 1201

Aracaju (SE)

Tel.: (79) 9977-9151

E-mail: carolprocto@hotmail.com 